\title{
Espaces d'Informations Personnelles : Utilisabilité et Modification Utilisateurs
}

\author{
CLAUDIA DETRAUX \\ Bertin Ergonomie, Montigny-le-Bretonneux \\ DOMINIQUE SCAPIN \\ INRIA Rocquencourt
}

Résumé : Cet article présente une étude sur l'utilisabilité d'un nouveau prototype de système de gestion des informations personnelles sur Internet (PIMI). Les objectifs sont d'évaluer sa facilité d'utilisation, et d'évaluer la modification utilisateur comme technique d'évaluation. Trente utilisateurs ont participé à l'expérience : une première partie consistait en un test utilisateur classique (TUC) et une seconde partie était un test d'utilisabilité avec modifications utilisateur (TUM). Un total de 51 problèmes d'utilisabilité a été diagnostiqué. Parmi eux, 32 ont été identifiés avec TUC, et 19 avec TUM. Une partie de ces derniers (11) s'ajoute à ceux identifiés avec TUC, et à ceux diagnostiqués précédemment lors d'une inspection de l'utilisabilité (IU avec Critères Ergonomiques). La participation active des utilisateurs au travers de scénarios de personnalisation semble fournir des indices supplémentaires pour l'évaluation de l'utilisabilité et pour la conception (nouvelles recommandations génériques d'utilisabilité).

Mots clés : Utilisabilité ; méthodes d'évaluation ; modifications utilisateur ; e-gov. ; informations personnelles.

Abstract: This paper presents a study on the usability of a new prototype system for managing personal information on the Internet (PIMI). The objectives are to assess its ease of use, and assess user modification as an evaluation technique. Thirty users participated in the experiment: the first part was a classical usability test (TUC) and a second part was a usability test with user modifications (TUM). A total of 51 usability problems were diagnosed. Among them, 32 with TUC, and 19 with TUM. Part of the latter (11) adds to those identified with TUC, and those previously diagnosed during a usability inspection (IU with Ergonomic Criteria). The active participation of users through customization scenarios seems to provide additional clues for evaluating usability and for design (new generic usability recommendations).

Key words: Usability ; evaluation methods ; user modifications ; e-government ; personal information.

\footnotetext{
Adresse des auteurs : Dominique Scapin (dominique.scapin@inria.fr), INRIA, Domaine de Voluceau, 78153 Le Chesnay Cedex, France. Claudia Detraux (claudia.detraux@bertin.fr), BERTIN Ergonomie, 10Bis Avenue Ampère, 78180 Montigny-le-Bretonneux, France.
}

Les articles de JIPS sont publiés sous licence Creative Commons Paternité 2.0 Générique. 


\section{INTRODUCTION}

Les PIMs (Personal Information Management systems) envahissent notre vie quotidienne, comme citoyens, consommateurs, employés, et notre cercle familial, amical, etc. Plusieurs types d'outils sont disponibles pour supporter ces activités et ainsi organiser les différentes données personnelles. Ces systèmes se doivent d'être utilisables, notamment pour atteindre l'objectif des initiatives gouvernementales de dématérialisation des procédures. Cette étude a été menée au sein du projet ANR-PIMI (Personal Information Management through Internet) dont l'objectif est l'élaboration d'un Espace d'Informations Personnelles (EIP) et de se doter de logiciels, dont un outil permettant de saisir, organiser et récupérer les informations dites personnelles. Cet EIP est géré à travers l'Internet et aidera à réaliser les différentes téléprocédures du type egouvernement (par exemple : demande d'un passeport, paiement des impôts, etc.).

Cet EIP est plus générique qu'un "profil client", ou que des formulaires spécifiques requis par les différentes procédures. Cet EIP appartient à l'utilisateur avec l'ensemble de ses données personnelles. Les autres formulaires pourront ainsi être un sousensemble de ces données avec des objectifs spécifiques (par exemple : demande de bourse étudiant, de passeport, de logement, paiement des impôts, etc.). Cet EIP pourra alimenter ces formulaires et "profils clients", et ainsi faciliter les différentes démarches et le remplissage.

Cette étude fait suite à un travail qui a contribué de manière itérative au prototype actuel. Plusieurs études d'utilisabilité ont été réalisées [Scapin et al. 2011] : une analyse de formulaires administratifs ; trois focus groups sur le stockage et le partage d'informations ; un questionnaire en ligne sur les mêmes thèmes pour élargir la population ; une étude de card-sorting pour valider les catégories crées par les utilisateurs et aussi leurs façons de les organiser. Une maquette PIMI a ensuite été élaborée. Une autre étude [Detraux and Scapin 2012] a complémenté cette approche par une expérimentation. Son objectif était de valider le contenu et la structure d'items d'information proposés au travers d'une maquette partiellement modifiable sur requête des utilisateurs. Les résultats ont permis de valider la structure d'items du futur espace personnel, de commencer à analyser les modifications suggérées par l'utilisateur comme indices d'évaluation et de proposer des recommandations pour améliorer son utilisabilité.

L'étude actuelle concerne l'évaluation ergonomique du nouveau prototype PIMI. Une expérimentation a été menée avec 30 participants. Elle se composait d'une partie test utilisateur classique (TUC) et d'une deuxième partie où les utilisateurs ont été invités à modifier le prototype (TUM). L'objectif était double : évaluer l'utilisabilité d'un prototype avancé de PIMI (contenu, structure et personnalisation), et étudier le rôle de différentes méthodes dans le diagnostic de problèmes d'utilisabilité. Les résultats du TUC et du TUM ont été comparés. Puis, ces résultats ont été confrontés à une Inspection de l'utilisabilité (IU) réalisée auparavant.

Cet article propose d'abord une revue bibliographique sur les PIMs, les interfaces modifiables, et la comparaison de méthodes d'évaluation de l'utilisabilité. Puis l'expérimentation est décrite (méthode, procédure, matériel, participants), ainsi que les principaux résultats obtenus. La discussion évoque les aspects méthodologiques et la conclusion résume les principaux enseignements et quelques pistes de recherche pour le futur. 


\section{TRAVAUX CONNEXES}

Personal Information Management systems (PIMs) : le domaine des systèmes de gestion de l'information personnelle se réfère notamment aux études sur la manière dont les utilisateurs gèrent leurs données et documents personnels. Du point de vue de l'utilisabilité, la littérature n'est pas encore très riche. Certains aspects sont couverts en partie dans les conférences de type CSCW [CSCW13-1,2013], bien sûr principalement sur la coopération, les groupes, les interactions sociales, et sur les aspects sociétaux dans [Oakley and Salam 2012]. D'autres articles sont plus directement concernés par les utilisateurs, comme «un écosystème numérique personnel de l'utilisateur» [Kay and Kummerfeld 2012], ou l'évolution de l'utilisation des tablettes [Buttfield-Addison et al. 2012], ou la vie privée [Ploderer et al. 2012] et [Rawassizadeh 2012]. Certaines contributions concernent la facilité de remplissage de formulaires dans [Zimmerman and Lorentz 2002], [Winckler et al. 2011], la recherche sur le web [Nitsche and Nuernberger 2012], et la flexibilité entre les périphériques [Ardito et al. 2012]. Certaines études longitudinales ont permis de mieux comprendre la prise de notes et l'archivage des informations [Van Kleek et al. 2011], ou les pratiques mobiles [Lindroth and Bergquist 2008]. Cependant, peu d'apports nouveaux sur l'évaluation de l'utilisabilité, par rapport à [Detraux and Scapin, 2012, op. cit.] ont été évoqués au delà des problèmes d'organisation et de récupération de l'information au sein des structures hiérarchiques, encore les plus répandues et préférées des utilisateurs [Evequoz and Lalanne 2009], [Henderson 2009], [Henderson and Srinivasan 2009], [Voit et al. 2009], mais qui montrent aussi les difficultés des utilisateurs avec la création de structures hiérarchiques cohérentes et significatives, ainsi qu'avec la dénomination des catégories et rubriques. L'expérience décrite ici tente d'aller plus loin sur ces questions de structure et de dénomination des informations personnelles.

Par ailleurs, nous avons analysé 15 outils ${ }^{1}$. La plupart (\#1, \#2, \#3, \#4, \#5, \#7, \#8, $\# 11, \# 12, \# 13, \# 15)$ proposent les fonctions suivantes : agenda, calendrier, contacts, recherche, gestionnaire des mots de passe, notes; la dénomination des concepts/items est un problème pour quelques outils $(\# 1, \# 4, \# 7)$; une structure d'information déjà établie est rarement proposée; l'outil (\#9) propose une structure à plusieurs niveaux de catégories/informations, et (\#1) et (\#14) présentent des structures simples de stockage des documents ; (\#9), (\#14) ne permettent pas la saisie de données, mais uniquement le chargement des fichiers; par ailleurs la plupart permettent de construire des catégories de notes/sous-notes, de tâches. Côté contenu, on constate notamment, à part pour (\#9), l'absence de structuration/format d'informations de type : identité, famille, santé, revenus, papiers d'identité, parcours professionnel, etc. Les interfaces sont assez similaires. Une personnalisation de l'interface est possible dans (\#1, \#3, \#8, \#10, \#15), dont les principaux paramètres modifiables sont : couleur, taille de la fenêtre, langue, position et affichage des menus / boutons, mais pas le contenu. L'expérience décrite ici englobe les aspects « contenu informationnel» (et structure).

Modifications Utilisateurs : un travail précédent [Detraux and Scapin 2012, op. cit.] a proposé une revue bibliographique sur les interfaces modifiables. Depuis, peu de

\footnotetext{
1 (\#1www.efficientpim.com, \#2 www.thebrain.com, \#3 www.essentialpim.com, \#4 www.winpim.com, \#5 www.lifemanagerpro.com, \#6 www.azzcardfile.com, \#7www.pimonline.com, \#8 www.pimone.com/pimone.htm, \#9 www.myarchivebox.com, \#10 www.evernote.com, \#11 http://code.google.com/p/keynote-nf, \#12 http://www.treepad.com, \#13 www.milenix.com, \#14 www.android-software.fr/pocket-docs, \#15 www.gmail.com)
} 
nouvelles contributions ont été identifiées au-delà de [Mørch and Mehandjiev 2000], [Zhu et al. 2011], [Demunieux et al. 2008], [Dörner et al. 2011] qui traitent de la modification utilisateur lors de la conception et le développement de systèmes (notamment les échanges entre utilisateurs et concepteurs), son utilisabilité et son impact sur les mesures d'utilisabilité. D'autres articles traitent plutôt de questions comme les systèmes de recommandations [Heung-Nam et al. 2013], réseaux sociaux [Zimmerman and Lorentz 2002], jeux [Gamberini et al. 2012], environnements physiques [Lindroth and Bergquist 2008], e-learning [Kolås and Staupe 2007], etc. En résumé, la modification a plutôt été utilisée jusqu'à maintenant dans la littérature pour aider la communication concepteurs-utilisateurs, mais pas comme moyen d'évaluation de l'utilisabilité. Le travail précédent à cette étude réalisée avec une maquette a permis déjà de constater le caractère participatif de cette démarche de modification, ce qui semble stimuler l'utilisateur futur. On constate que la possibilité de personnaliser l'interface aide à lever le blocage participatif dans un test utilisateur, ce qui peut aider dans un processus d'évaluation de l'utilisabilité.

Cette étude tente d'apporter de nouveaux résultats empiriques sur ce point. Pour cela, le principe a été de proposer à des utilisateurs d'effectuer eux-mêmes les modifications qu'ils souhaitaient sur le contenu et la structure du prototype, à leur guise ou selon un scénario.

Méthodes d'Évaluation de l'Utilisabilité : de nombreuses méthodes sont proposées dans la littérature (voir [Scapin et al. 2008] et (pour Test Utilisateurs et Inspection) voir ISO 16982 [ISO/TR 16982. 2002], notamment 5.1 Methods which imply the direct involvement of users, et 5.2 Methods which do not imply the direct involvement of users. Leur comparaison est longue et complexe, en particulier lorsque l'on suit des lignes directrices et critères tels que dans [Gray and Salzman 1998], [Hartson, et al. 2001]. Lors d'un examen approfondi de la littérature [Bach and Scapin 2010] diverses questions ont été rapportées (voir [Cockton et al. 2003], [Gray and Salzman 1998], [Hartson et al 2001], [Hornbæk and Frøkjær 2005]), comme l'utilisation de mesures appropriées, la gravité des problèmes d'utilisabilité, leur authenticité, la rigueur des analyses, les problèmes d'interprétation des problèmes et leur similitude. Dans cette étude, nous avons tenté de pallier ces difficultés grâce à un soin particulier au codage de problèmes. Cependant, alors que nous avons utilisé un assez grand nombre de participants pour l'expérience, l'inspection ergonomique a, quant à elle, été coréalisée par seulement 2 experts, contrairement à l'étude citée [Bach and Scapin 2010, op. cit.].

\section{EXPERIMENTATION}

L'objectif de l'expérimentation est d'évaluer le prototype PIMI en obtenant des informations sur le comportement intuitif de l'utilisateur. Les objectifs spécifiques sont de : (1) vérifier l'utilisabilité de l'interface du système pour saisir des informations, retrouver des items, et personnaliser la dénomination des catégories, des rubriques, changer l'ordre d'affichage, masquer des catégories et rubriques; (2) vérifier si la dénomination des catégories, rubriques et items est compréhensible et convient aux utilisateurs ; (3) vérifier si la structure proposée est compréhensible et acceptée par les utilisateurs ; (4) vérifier si les utilisateurs souhaitent modifier leurs espaces personnels ; et conjointement vérifier si les scénarios de modification apportent des indices additionnels à l'évaluation ergonomique, par rapport aux scénarios classiques. 
Les mesures étaient répétées (plan intra); les variables indépendantes étant les caractéristiques des participants; et la variable dépendante étant le comportement des sujets (temps, difficultés, erreurs, etc.). Le prototype était le même pour tous les participants et les tâches. L'étude étant qualitative, l'analyse a porté essentiellement sur un examen détaillé des opérations effectuées (nature des modifications), les données se prêtant mal à des statistiques inférentielles, à cette étape.

Méthode et procédure : Les séances ont été réalisées individuellement et accompagnées par l'expérimentateur qui a présenté le projet, expliqué les objectifs de l'expérimentation et le prototype (comment naviguer, saisir, enregistrer et modifier le PIMI) ; questionné les participants ; les a incités et motivés à parler de leurs difficultés en rapport aux tâches, à l'outil, aux données. De leur côté, les participants devaient réaliser les tâches demandées par l'expérimentateur, répondre aux questionnaires et exprimer leur opinion. Pour mettre les participants en situation, on a choisi de mener les séances dans leur propre environnement de travail (dans leurs bureaux pour les chercheurs et le personnel administratif) ou d'étude (salle d'informatique pour les étudiants). De cette façon, ils pouvaient accéder facilement à leurs effets personnels (sac, portefeuilles, agendas, ordinateurs personnels ou de travail), pour la réalisation des tâches demandées. Le prototype a été présenté sur un ordinateur portable et les séances enregistrées avec un logiciel de capture d'écran (vidéo et son).

Un test-pilote a été réalisé avec un sujet de formation ingénieur. Cela a permis de valider la consigne et le discours de l'expérimentateur ainsi que de calibrer la durée des sessions. Les séances se sont déroulées en 3 phases principales :

- Présentation du projet et de l'application (comment naviguer, saisir les données et effectuer les modifications). Les participants sont invités à penser à voix haute pendant l'exécution des tâches.

- Travail sur Scénarios : Dans une première partie, 4 scénarios ont été proposés consécutivement :

Scénario 1 : tâches d'identification personnelle (saisir nom, prénom, informations de la carte d'identité, adresse, téléphone). L'objectif est de laisser l'utilisateur se familiariser avec l'outil, naviguer, découvrir son contenu et commencer à se l'approprier;

Scénario 2: tâches qui concernent les contacts professionnels et le parcours étudiant; L'objectif est de vérifier si les utilisateurs trouvent facilement les rubriques dans la structure, s'ils arrivent à bien naviguer, sauvegarder les données saisies et à charger des documents. Dans les travaux précédents la classification de ces rubriques et items a identifié quelques conflits ([Scapin et al. 2011], [Detraux and Scapin 2012]).

Scénario 3 : tâches de saisie dans 2 types de formulaires. L'objectif est de vérifier si les utilisateurs comprennent leurs différences, et s'ils ont des difficultés à retrouver les informations;

Scénario 4 : tâches destinées à vérifier (chemin parcouru) si les redondances proposées sont comprises et utilisées.

Dans une deuxième partie, un des objectifs était d'étudier le caractère modifiable d'un système de ce type par les utilisateurs afin d'adapter leur espace personnel selon leurs besoins et envies. Deux étapes sont proposées : 
Étape 1 (Modifications par consigne): scénario avec consignes expérimentales de modifications, afin de surtout vérifier la facilité à les réaliser et le(s) chemin(s) utilisés pour les effectuer (fonction «Personnaliser le PIMI» ou «Options de Personnalisation » de « Saisir et Visualiser les Informations »).

Étape 2 (Modifications à l'initiative des participants) : scénario demandant de parcourir toute la structure du PIMI et d'effectuer des modifications selon les souhaits des participants.

Finalement dans une troisième partie, les participants ont répondu à un questionnaire composé de 2 sections :

- La première section comportait des questions spécifiques sur l'aspect modification, pour comprendre l'acceptation de cette étape ainsi que les préférences des utilisateurs pour ce type de modification, ainsi que les difficultés à la réaliser.

- La deuxième section comportait des questions plus générales sur le système PIMI et sur les améliorations souhaitées. Les questions sont «fermées» (oui/non ou liste d'options). Cependant, l'expérimentateur encourage l'utilisateur à justifier ses réponses oralement (enregistrement audio) ou par écrit.

Participants : Les séances ont été réalisées avec 30 participants (10 chercheurs, 10 personnels administratifs et 10 étudiants), dont 16 participants masculins $(53,3 \%)$ et 14 participants féminins (46,6 \%). La moyenne d'âge est de 36,06 ans (sd. 16,8).

Dans la catégorie «chercheurs », 8/10 des participants sont masculins (80\%), et 2/10 féminins (20\%). La moyenne d'âge est de 47,5 ans (sd. 17,48).

Dans la catégorie «personnel administratif », 9/10 des participants sont féminins (90\%), et 1/10 masculin. La moyenne d'âge est de 41,2 ans (sd. 11,4).

Dans la catégorie «étudiants », 7/10 participants sont masculins (70\%) et 3 participants féminins (30\%). La moyenne d'âge est de 19,5 ans (sd. 1,17).

\section{Matériel :}

Items d'Information: Les résultats des études précédentes [Detraux et Scapin 2012] ont abouti à une structure de 73 items d'information, avec 10 catégories, et 35 rubriques (voir Tableau 1).

Matériel Expérimentateur : Un logiciel de capture vidéo ( $\mathrm{NCH}$ Software) permet d'enregistrer en vidéo (écran) les séances ainsi que les commentaires des participants pendant l'exécution des scénarios (avec la permission des utilisateurs). Des check-lists sont également remplies par l'expérimentateur afin de noter les informations les plus pertinentes, et les évènements importants.

Matériel Participants : Ordinateur portable avec prototype / logiciel de capture installé ; fiches de description des scénarios et tâches à accomplir ; fiches additionnelles pour les cas où l'utilisateur n'a pas sur lui les données à saisir (l'expérimentateur lui fournit une fiche qui simule une carte de visite d'un médecin spécialiste, d'un collègue, etc.) ; fichiers à charger qui sont demandés dans les scénarios, comme : carte d'identité fictive.jpg, parcours universitaire fictif.pdf.; questionnaire final comportant des questions sur l'application, son utilisation et le degré d'adoption d'un tel système. Ce 
questionnaire comporte une partie spécifique sur la modification et la participation utilisateurs.

\section{Nouvelle Structure}

\section{Identité}

- Identité \& Coordonnées personnelles

- Pièces d'Identité (Redondance avec Papiers)

2. Travail

- Travail actuel \& Coord. pro.

- Parcours professionnel

- Parcours étudiant

- Contacts pro. (Redondance avec Contacts)

3. Papiers

- Pièces d'Identité (Redondance avec Identité)

- Bulletins de salaire

- Carte vitale

\section{Contacts}

- Contacts personnels

- Contacts pro. (Redondance avec Travail)

5. Agenda

- Agenda personnel

- Agenda professionnel

6. Transports

- Transport individuel

- Transport collectif

7. Codes :

- Codes d'entrée

- Codes de verrouillage

- Codes de téléphones

- Mots de passe d'accès

- Codes site internet

- Codes carte paiement

8. Finances :

- Revenus \& allocations

- Placements

- Emprunts

- Compte(s) bancaire(s)

9. Impôts

- Revenu fiscal de référence

- Total de réduction d'impôts

- Tranche d'imposition

- Taxe foncière

- Taxe habitation

10. Santé

- Sécurité sociale \& Mutuelle

- Médecins

- Dossier Médical

- Personne à contacter en cas d'urgence

Tableau 1 : Catégories et Rubriques 
Prototype PIMI : Le prototype évalué (V 0.3, version PC) a été mis à disposition localement et accédé par le navigateur Firefox sur une plateforme Windows. Deux fonctions principales étaient disponibles pour les tests :

- «Saisir/visualiser les informations» : permet à l'utilisateur de visualiser l'espace personnel d'information (catégories/rubriques et items d'information), de saisir ses informations dans l'espace ainsi que de les éditer/corriger.

- "Personnaliser le PIMI » : permet de changer les dénominations, l'ordre d'affichage, de masquer catégories et rubriques.

Les Figures suivantes montrent des images capturées du prototype.

e-Citiz PIMI

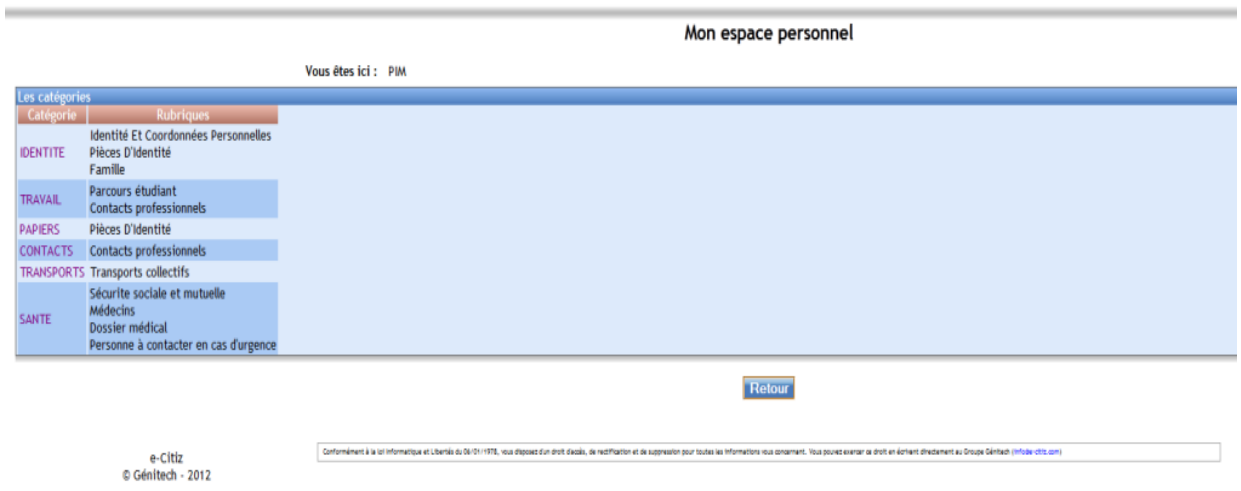

Figure 1. Écran du prototype PIMI (Mon espace personnel)

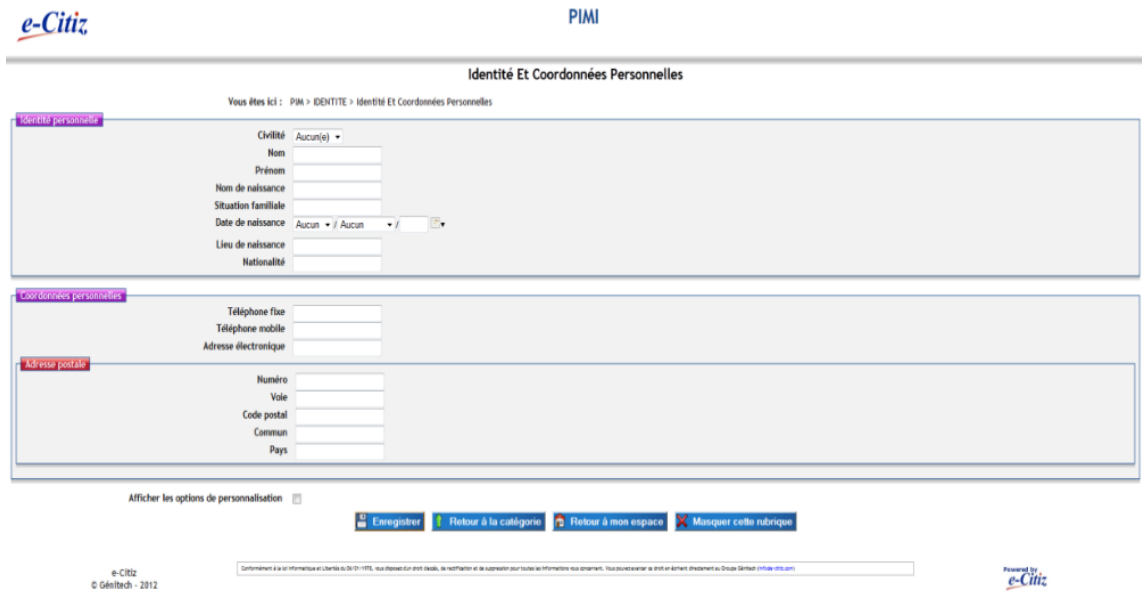

Figure 2. Écran du prototype PIMI (Identité et Coordonnées personnelles) 


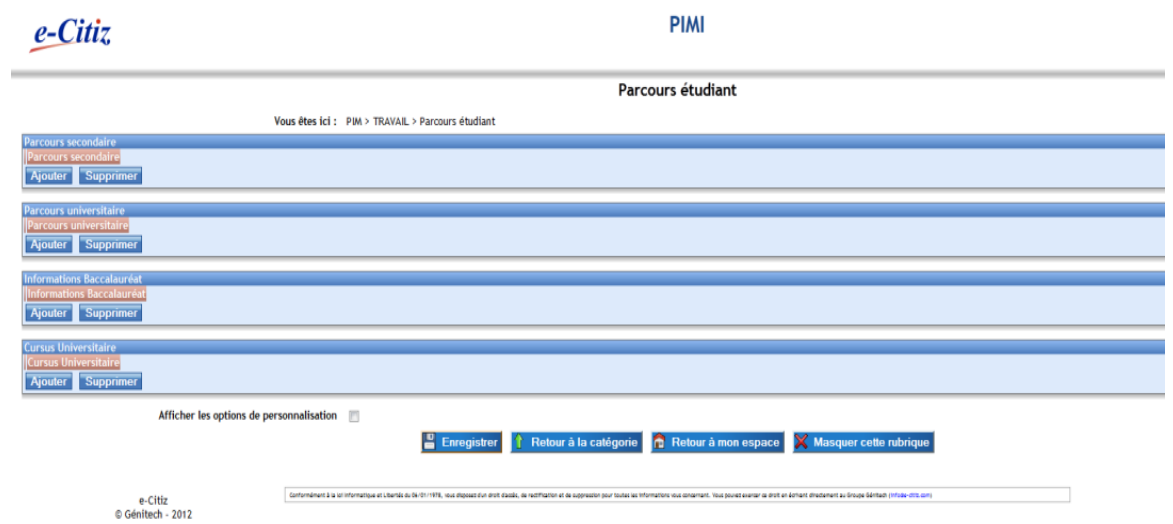

Figure 3. Écran du prototype PIMI (Parcours étudiant)

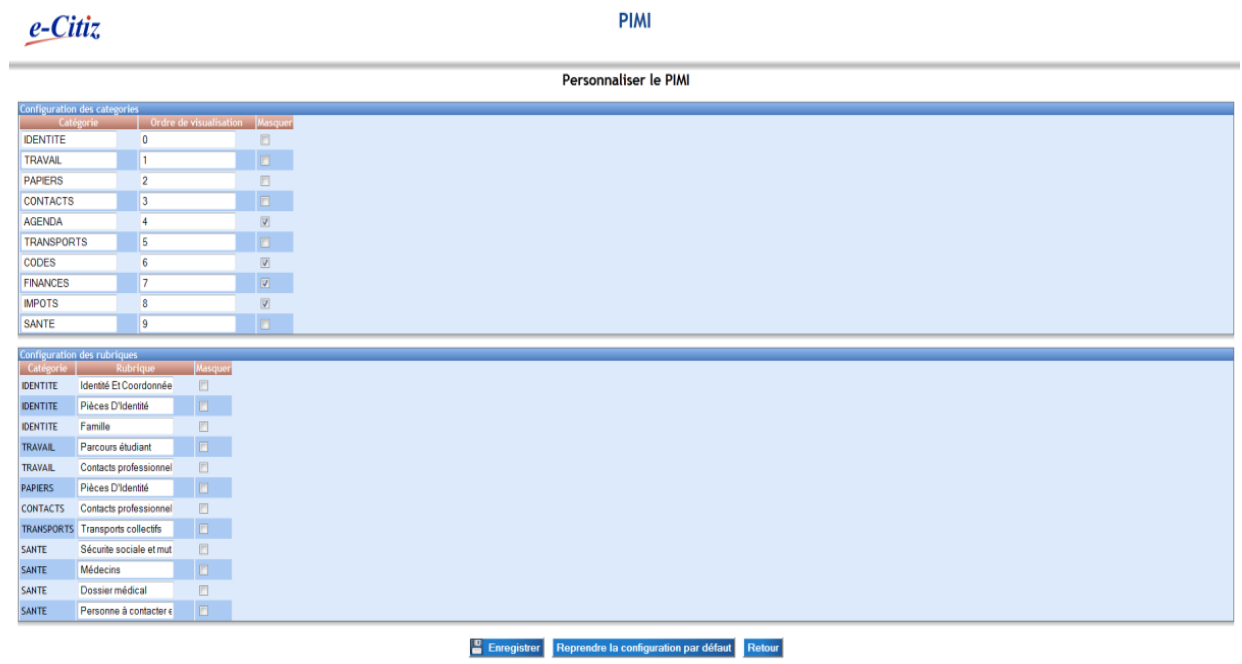

Figure 4. Écran du prototype PIMI (Personnaliser le PIMI)

\section{RESULTATS}

Cette partie présente les résultats de l'expérimentation. Nous avons réalisé au total 30 séances avec utilisateur. Tous les participants ont réalisé toutes les étapes et ont autorisé l'enregistrement.

Durée des séances : la durée moyenne a été de 46,93 minutes (sd. 10,16). Dans la catégorie «chercheurs", la moyenne est de 46,3 minutes (sd. 8,38) ; pour les 
«personnels administratifs » de 51,9 minutes (sd. 11,76) ; et pour les «étudiants » de 42,6 minutes (sd. 8,69).

Les analyses sont basées sur les «difficultés » et les «erreurs », ainsi que sur les commentaires des participants. Les difficultés englobent les délais pour trouver une information, une navigation confuse, un temps d'inactivité ; les erreurs sont des actions erronées ou sans lien ; les commentaires sont exprimés par les participants pendant les séances ou lors du questionnaire. La plupart des commentaires renforcent des difficultés ou erreurs observées. On peut remarquer que les problèmes ont été détectés à partir des erreurs et difficultés observées (33\%), des commentaires des participants (27\%), et $40 \%$ par les deux moyens (voir la discussion qui détaille cet aspect).

Groupes de participants (voir Tableau 2) : on constate, pour TUC, des nombres d'erreurs similaires. Pour TUM, en termes de modifications, les étudiants ont plus modifié (19 dénominations, 37 masquages et 34 ordres, pour un total de 90), tandis que les administratifs en ont effectué moins (16 dénominations, 32 masquages, et 34 ordres, pour un total de 75), et les chercheurs encore moins 50 (7 dénominations, 34 masquages, et 9 ordres, pour un total de 50).

\begin{tabular}{|c|c|c|c|}
\cline { 2 - 4 } \multicolumn{1}{c|}{} & Chercheurs & Administratifs & Étudiants \\
\hline Erreurs TUC & $39 / 124$ & $42 / 124$ & $43 / 124$ \\
\hline Modifs TUM & 50 & 75 & 90 \\
\hline
\end{tabular}

Tableau 2 : Erreurs et modifications par groupe

Globalement, il semble que les utilisateurs les plus jeunes (étudiants) inclinent plus à proposer et adapter leur espace personnel.

Les réponses au questionnaire ont d'ailleurs montré que les seuls avis négatifs sur la facilité d'adaptation ( 5 participants) viennent de 3 chercheurs et de 2 administratifs.

Difficultés à trouver des informations L'accès aux catégories et souscatégories n'est pas vraiment un problème, contrairement à trouver certains items individuels (voir Tableau 3).

\begin{tabular}{|c|c|c|}
\cline { 2 - 3 } \multicolumn{1}{c|}{} & Difficultés & Items \\
\hline Scénario 1 & $16,1 \%$ & Parents \\
\hline Scénario 2 & $3,2 \%$ & Contacts professionnels \\
\hline Scénario 3 & $19,4 \%$ & $\begin{array}{c}\text { Sécurité sociale, médecin spécialiste } \\
\text { (et 58,1\% n'ont pas trouvé) }\end{array}$ \\
\hline Scénario 4 & $33,3 \%$ & $\begin{array}{c}\text { «ID» (fichier joint) } \\
\text { (et 3,3\% n'ont pas trouvé) }\end{array}$ \\
\hline
\end{tabular}

Tableau 3 : Difficultés à trouver des informations

Problèmes de dénomination. Les termes avec lesquels les participants ont eu quelques difficultés de compréhension (voir Tableau 4) semblent correspondre à des termes mal choisis, ou manquant de précision, ou encore présentés sans un contexte suffisant. 


\begin{tabular}{|c|c|c|}
\hline Rubrique & Difficultés & Items \\
\hline Identifications personnelles & 6 & Situation familiale \\
& 3 & Nom de naissance \\
& 1 & Lieu de naissance \\
\hline Informations collègues & 11 & Numéro \\
\hline Cursus de l'étudiant & 2 & Cursus universitaire et cours \\
\hline Sécurité sociale & 8 & Bénéficiaire \\
& 25 & Période de couverture \\
& 11 & Nombre \\
\hline Autres spécialistes de santé & 28 & Nom personnalisé \\
\hline
\end{tabular}

Tableau 4 : Difficultés liées à la dénomination

Difficultés de navigation (voir Tableau 5) : 19/30 participants ont eu au moins une difficulté, un seul en a eu 4 (total 33, moyenne 1,1; sd. 1,15). Les problèmes d'utilisabilité sont principalement liés à certains boutons de navigation (y compris libellés) et au manque de raccourcis de navigation.

\begin{tabular}{|c|c|c|}
\cline { 2 - 3 } \multicolumn{1}{c|}{} & Difficultés & Erreurs \\
\hline Scénario 1 & 7 & 3 \\
\hline Scénario 2 & 5 & 2 \\
\hline Scénario 3 & 4 & 2 \\
\hline Scénario 4 & 5 & 1 \\
\hline Modifs 1 & 7 & 3 \\
\hline Modifs 2 & 5 & 4 \\
\hline
\end{tabular}

Tableau 5 : Difficultés de navigation

Enregistrement de formulaires (voir Tableau 6). Cette opération donne lieu à 30 difficultés : 70\% des participants ont eu au moins 1 difficulté, et 3 participants ont eu 3 difficultés. Les problèmes d'utilisabilité sont principalement liés au manque de feedback, et à des confusions de dénomination.

\begin{tabular}{|c|c|c|}
\cline { 2 - 3 } \multicolumn{1}{c|}{} & Difficultés & Erreurs \\
\hline Scénario 1 & 13 & 2 \\
\hline Scénario 2 & 7 & 4 \\
\hline Scénario 3 & 9 & 2 \\
\hline Scénario 4 & \multicolumn{2}{|c|}{ Non concerné } \\
\hline Modifs 1 & 0 & 0 \\
\hline Modifs 2 & 0 & 1 \\
\hline
\end{tabular}

Tableau 6 : Difficultés d'enregistrement de formulaires

Globalement, les erreurs confirment les constatations précédentes. Un total de 124 erreurs a été observé lors de l'expérience (moyenne 4,13 par participant, sd. 2,17) : Scénario 1 (moyenne 1,13 , sd. 1,25), avec au moins 1 erreur pour 18 participants, et 5 pour 1 participant ; Scénario 2 (moyenne 1,3, sd. 1,2), avec au moins 1 erreur pour 20 participants et 4 pour 1 participant ; Scénario 3 (moyenne 1,3, sd. 1,05), avec au moins 1 
erreur pour 23 participants et 5 pour 1 participant ; Scénario 4 : seulement 1 erreur ; Scénario $5.1: 3$ erreurs pour 3 participants (1 chacun) ; Scénario $5.2: 8$ erreurs (moyenne 0,26 ; sd. 0,69), avec au moins 1 erreur pour 5 participants, et 3 pour 1 .

\section{Personnalisation : Modifications par consigne}

Chemins parcourus : pour le changement de dénomination: 63,3\% des participants (19/30) sont partis directement à la fonction "Personnaliser le PIMI» tandis que $6,7 \%$ des participants $(2 / 30)$ ont choisi d'aller dans chaque rubrique. Les autres $30 \%$ des participants (9/30) ont utilisé les deux chemins (d'abord par les rubriques et ensuite par la fonction «Personnaliser le PIMI »).

\section{Difficultés :}

- Pour le changement des dénominations : $90 \%$ (27/30 participants) ont effectué facilement les modifications, 2 participants $(6,7 \%)$ ont pris un peu de temps (>30 s.) pour les réaliser, et seulement $1(3,3 \%)$ n'a pas réussi.

- Pour le masquage : 56,7 \% des participants (17/30) ont effectué facilement le masquage des catégories et rubriques, 11 participants $(36,7 \%)$ ont pris un peu de temps pour le réaliser, et $2(6,7 \%)$ n'ont pas réussi.

- Pour le changement d'ordre : 43,3 \% des participants (13/30) ont effectué facilement le masquage des catégories et rubriques, et 17 participants $(36,7 \%)$ ont pris un peu de temps pour le réaliser.

- Pour la restauration de la structure par défaut : 96,7\% des participants (29/30) ont effectué facilement la restauration par défaut de la structure, 1 seul participant $(3,3 \%)$ ayant pris un peu de temps pour la réaliser.

Personnalisation : Modifications à l'initiative des participants : Le Tableau 7 montre un total de 222 modifications suggérées (Chercheurs $\mathrm{C} 1$ à $\mathrm{C} 10$; Administratifs $\mathrm{A} 1$ à A10 ; Étudiants, E1 à E10), avec une moyenne de 7,4 par participant (sd. 4,79).

Tous les participants ont effectué des modifications. La personne qui a fait le plus de modifications en a suggéré 19 alors que 4 personnes en ont suggéré seulement 1 . Le Tableau 7 montre que le type de modification le plus fréquent est le masquage (catégories, rubriques et items) : (103 modifications), suivi du changement d'ordre des catégories (77 modifications), puis le changement des dénominations (42 modifications).

- Pour le masquage, 58 modifications ont concerné les catégories (93,3\% participants), 22 les rubriques (14,3\% participants), et 23 les items (3,2\% participants). Les raisons pour lesquelles les utilisateurs ont effectué ce type de modification sont : le besoin de personnalisation/simplification de l'Espace Personnel, ils laissent les catégories, rubriques qu'ils pensent utiliser; le besoin de sécuriser / protéger les données. Ils remplissent leurs informations, mais ensuite ils les «cachent» (en les masquant) : " ... si j’ai besoin de la visualiser, je sais où je peux la démasquer, mais comme ça elle n'est pas visible ». C'est le cas pour des rubriques comme Dossier Médical, Famille. 


\begin{tabular}{|c|c|c|c|c|c|c|c|}
\hline & $\begin{array}{c}\text { Dénomination } \\
\text { Catégories }\end{array}$ & $\begin{array}{c}\text { Dénomination } \\
\text { Rubriques }\end{array}$ & $\begin{array}{l}\text { Masquage } \\
\text { Catégories }\end{array}$ & $\begin{array}{l}\text { Masquage } \\
\text { Rubriques }\end{array}$ & $\begin{array}{c}\begin{array}{c}\text { Masquage } \\
\text { Items }\end{array} \\
\end{array}$ & $\begin{array}{c}\text { Ordre } \\
\text { catégories }\end{array}$ & Total \\
\hline $\mathrm{C} 1$ & 0 & 3 & 1 & 1 & 1 & 0 & 6 \\
\hline $\mathrm{C} 2$ & 1 & 0 & 1 & 0 & 0 & 0 & 2 \\
\hline C3 & 0 & 0 & 2 & 3 & 0 & 0 & 5 \\
\hline C4 & 0 & 2 & 1 & 2 & 0 & 4 & 9 \\
\hline C5 & 0 & 0 & 5 & 2 & 0 & 3 & 10 \\
\hline C6 & 0 & 1 & 0 & 0 & 0 & 0 & 1 \\
\hline C7 & 0 & 0 & 2 & 0 & 5 & 0 & 7 \\
\hline $\mathrm{CB}$ & 0 & 0 & 1 & 0 & 0 & 0 & 1 \\
\hline $\mathrm{CP}$ & 0 & 0 & 3 & 1 & 0 & 2 & 6 \\
\hline $\mathrm{C} 10$ & 0 & 0 & 2 & 1 & 0 & 0 & 3 \\
\hline A1 & 0 & 0 & 0 & 0 & 0 & 5 & 5 \\
\hline A2 & 4 & 1 & 4 & 2 & 0 & 0 & 11 \\
\hline $\mathrm{A} 3$ & 1 & 2 & 3 & 2 & 0 & 9 & 17 \\
\hline A4 & 0 & 0 & 1 & 0 & 0 & 4 & 5 \\
\hline A5 & 2 & 0 & 3 & 0 & 0 & 8 & 13 \\
\hline A6 & 2 & 1 & 1 & 0 & 0 & 4 & 8 \\
\hline A7 & 0 & 0 & 4 & 0 & 0 & 0 & 4 \\
\hline A8 & 2 & 1 & 1 & 0 & 0 & 4 & 8 \\
\hline A9 & 0 & 0 & 1 & 0 & 0 & 0 & 1 \\
\hline A10 & 0 & 0 & 4 & 0 & 6 & 0 & 10 \\
\hline E1 & 1 & 0 & 2 & 0 & 5 & 0 & 8 \\
\hline E2 & 3 & 2 & 1 & 1 & 0 & 0 & 7 \\
\hline E3 & 2 & 0 & 1 & 1 & 0 & 2 & 6 \\
\hline E4 & 0 & 0 & 1 & 0 & 0 & 0 & 1 \\
\hline E5 & 0 & 0 & 1 & 0 & 0 & 8 & 9 \\
\hline E6 & 1 & 1 & 2 & 2 & 6 & 4 & 16 \\
\hline E7 & 0 & 0 & 1 & 0 & 0 & 4 & 5 \\
\hline E8 & 0 & 1 & 3 & 1 & 0 & 0 & 5 \\
\hline E9 & 2 & 3 & 5 & 2 & 0 & 7 & 19 \\
\hline E10 & 1 & 2 & 1 & 1 & 0 & 9 & 14 \\
\hline TOTAL & 22 & 20 & 58 & 22 & 23 & 77 & 222 \\
\hline MOYENNE & 0,73 & 0,66 & 1,93 & 0,73 & 0,76 & 2,56 & 7,4 \\
\hline ECART-TYPE & 1,08 & 0,95 & 1,38 & 0,90 & 1,90 & 3,10 & 4,79 \\
\hline
\end{tabular}

Tableau 7 : Modifications par groupes de participants

- Pour les modifications d'ordre, $50 \%$ des participants ont effectué au moins 2 modifications (maximum 9). Les participants veulent surtout classer les catégories selon leur utilisation et leur importance. Ces deux critères sont assez personnels, par exemple nous montrons ci-dessous (Figure 5) 3 organisations différentes proposées par les utilisateurs. 


\begin{tabular}{|c|c|}
\hline \multicolumn{2}{|c|}{ Configuration des categories } \\
\hline Catégorie & Ordre de visualisation \\
\hline IDENTITE & 0 \\
\hline TRAVAll & 1 \\
\hline PAPIERS & 2 \\
\hline SANTE & 3 \\
\hline CODES & 4 \\
\hline TRANSPORTS & 5 \\
\hline AGENDA & 6 \\
\hline FINANCES & 7 \\
\hline CONTACTS & 8 \\
\hline IMPOTS & 9 \\
\hline
\end{tabular}

\begin{tabular}{|l|l|}
\hline \multicolumn{1}{|c|}{ Configuration des categories } \\
\cline { 1 - 1 } & \multicolumn{1}{|c|}{ Ordre de visualisation } \\
\hline IDENTITE & 0 \\
\hline TRAVAILIETUDES & 1 \\
\hline PAPIERS & 2 \\
\hline CONTACTS & 3 \\
\hline AGENDA & 4 \\
\hline TRANSPORTS & 5 \\
\hline CODES & 6 \\
\hline FINANCES & 7 \\
\hline IMPOTS & 8 \\
\hline SANTE & 9 \\
\hline
\end{tabular}

\begin{tabular}{|c|c|}
\hline \multicolumn{2}{|c|}{ Configuration des categories } \\
\hline Catégorie & Ordre de visualisatior \\
\hline IDENTITE & 0 \\
\hline SANTE & 1 \\
\hline TRAVAll. & 2 \\
\hline CONTACTS & 3 \\
\hline PAPIERS & 4 \\
\hline CODES & 6 \\
\hline FINANCES & 7 \\
\hline IMPOTS & 8 \\
\hline AGENDA & 8 \\
\hline TRANSPORTS & 9 \\
\hline
\end{tabular}

Figure 5 : Modifications d'ordre

Malgré la difficulté à faire des changements d'ordre des catégories, les utilisateurs sont initialement très motivés, mais plusieurs entre eux ont été freinés par la difficulté de le faire en n'arrivant pas à gérer la numérotation.

- Pour le changement de dénomination, les principales raisons sont: le manque de précision dans la dénomination des catégories et rubriques et le besoin de simplifier les dénominations.

Enfin, $47 \%$ des participants ont exprimé le besoin d'un ensemble de modifications non proposées : ajout de catégories ou sous-catégories $(33 \%)$; division d'une catégorie $(0,6 \%)$, déplacement de sous-catégories $(0,6 \%)$; distinction entre entrée de données et récupération de données $(0,3 \%)$, modification des codes couleur, de la taille des caractères $(0,6 \%)$.

\section{Questionnaire}

Section 1 : questions sur la personnalisation : La plupart des participants (83.3\%) ont apprécié de pouvoir modifier leur Espace Personnel d'information. La plupart $(93,3 \%)$ ont également trouvé qu'il était facile de modifier les noms des catégories et des sous-catégories $(80 \%)$. Il leur a également semblé facile de masquer des catégories et sous-catégories $(93,3 \%)$. Il a été jugé beaucoup moins facile de modifier l'ordre des catégories $(36,7 \%)$. Les utilisateurs ont quand même suggéré des solutions et des idées pour améliorer les modes d'effectuer les modifications. En outre, les participants ont suggéré d'autres types de modifications qui n'ont pas été mises en œuvre dans le prototype, telles que : ajouter de nouvelles catégories et sous-catégories (9/20); changer le design visuel, par exemple, les couleurs, les formats $(4 / 30)$; et fournir les formulaires horizontalement $(2 / 30)$.

Section 2: questions générales et améliorations : Une courte majorité des participants $(60 \%)$ se sent prêt à utiliser un tel PIMI. Leur motivation est essentiellement la capacité à centraliser toutes leurs données et de limiter la consommation de papier. Pour ceux qui se sentent moins prêts $(33,3 \%)$ et ceux qui n'étaient pas prêts du tout $(6,7 \%)$, les principales préoccupations sont la sécurité et la confiance. La plupart des participants $(80 \%)$ ont dit ne pas avoir éprouvé de difficultés pour utiliser le prototype. Seules ont été exprimées quelques difficultés modérées $(16,7 \%)$ ou fortes $(3,3 \%)$. En 
outre, 25/30 participants $(86,2 \%)$ ont fait des suggestions d'amélioration, essentiellement pour atténuer certains des problèmes d'utilisabilité mentionnés précédemment.

D'un point de vue méthodologique, il est à remarquer que, globalement, les diverses évaluations d'utilisabilité menées dans cette étude ont conduit au diagnostic d'un plus grand ensemble de problèmes d'utilisabilité que les seules réponses au questionnaire. Toutefois, celles-ci, en particulier les suggestions, ont confirmé une partie des problèmes identifiés.

\section{DISCUSSION}

Personnalisation : La participation lors de cette session a été forte : un total de 222 modifications ont été apportées, et tous les participants ont fait au moins un changement avec un maximum de 19 pour un participant (moyenne 7,4 ; sd. 4,79).

D’une façon générale, les participants ont aimé pouvoir modifier et personnaliser leur PIMI et ils ont trouvé facile d'effectuer les modifications. Les modifications peuvent être caractérisées selon 4 buts principaux : simplifier (par masquage et changement de dénomination), clarifier (par changement de nom), faciliter l'accès (par changement d'ordre des catégories); sécuriser (par masquage).

On a pu détecter des problèmes non détectés auparavant :

- La nécessité claire d'avoir un vocabulaire qui puisse s'adapter aux différents profils d'utilisateurs; les besoins et la vision des plus jeunes (étudiants) sont vraiment différents de ceux qui sont déjà dans la vie professionnelle.

- L'envie d'avoir d'autres catégories et rubriques : l'utilisateur souhaite aussi créer des nouvelles catégories ou structures pour organiser l'espace et mettre des informations additionnelles.

- Le besoin de sécuriser, cacher les informations : une possibilité additionnelle donnée par les utilisateurs à la fonction masquage des rubriques et catégories. On s'aperçoit de la nécessité de proposer à l'utilisateur un système paramétrable où il puisse choisir à qui montrer ses données (famille, amis, administration, autres).

On a pu renforcer d'autres problèmes détectés auparavant, mais pas encore mentionnés explicitement par l'utilisateur :

- Changer le visuel de l'interface : les couleurs, les tailles de police et ainsi pouvoir mieux identifier les informations,

- Uniformiser les formulaires en ligne

- Le mode de changement d'ordre des catégories doit être simplifié

- Un arbre avec toute la structure modifiable (catégories, rubriques et items).

Quelques enseignements méthodologiques peuvent être tirés des modifications des participants :

- Cela permet d'identifier des problèmes d'utilisabilité supplémentaires.

- Les participants ont tendance à considérer leur rôle comme plus important, à s'exprimer plus, à fournir des critiques, des suggestions et des préférences. En bref, ils s'approprient le système, voire, renforcent leur opinion sur certains problèmes d'utilisabilité qui ne semblent pas trop "gênants" initialement.

- Compte tenu de la nécessité pour les systèmes actuels d'e-gouvernement d'être utilisés par un public large et varié en termes d'âge, de sexe, de profession, de 
compétences et de contextes, il est utile de fournir des fonctions de personnalisation par les utilisateurs (par exemple, pour le vocabulaire, les techniques, les besoins de navigation).

En résumé, faciliter les utilisateurs dans un rôle d'acteurs plutôt que d'utilisateurs passifs semble prometteur.

Comparaison de méthodes d'évaluation de l'utilisabilité : une comparaison entre les différentes méthodes d'évaluation utilisées a été réalisée : l'Inspection Ergonomique avec les Critères Ergonomiques, le Test Utilisateur avec des scénarios classiques et le Test Utilisateur avec scénarios de modification.

La Figure 6 montre le nombre de problèmes d'utilisabilité diagnostiqués avec les 3 méthodes d'utilisabilité (test d'utilisabilité classique (TUC), test d'utilisabilité avec modifications (TUM) et inspection de l'utilisabilité (IU).

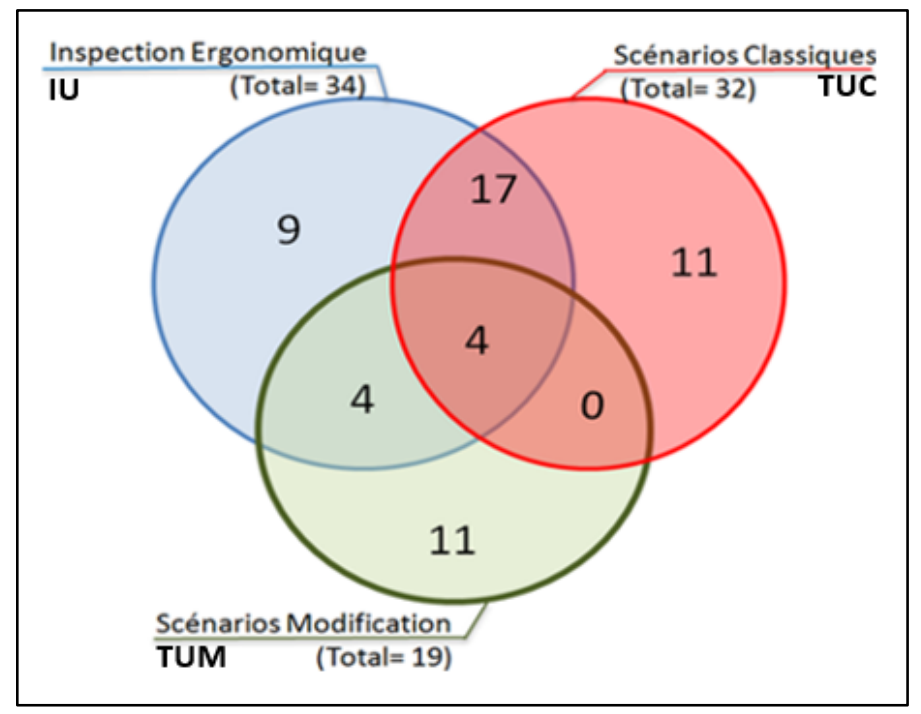

Figure 6 - Problèmes d'utilisabilité par méthode

Globalement, il n'y a pas de différences marquantes dans la nature des problèmes d'utilisabilité, si ce n'est, évidemment, celles liées aux différents états d'interaction parcourus, du fait des scénarios de tâches (états de modification pour TUM et états de saisies de données pour TUC). Toutefois, on trouve :

- Environ le même nombre de problèmes d'utilisabilité spécifiques pour chaque technique d'évaluation : 9 pour IU ( $26 \%$ du total) ; 11 pour TUC (34\% du total), 11 pour TUM (58\% du total). Cependant, en pourcentage, TUM a le score relatif le plus élevé de problèmes spécifiques.

- Beaucoup de problèmes communs (meilleur score) à IU et TUC $(21$, soit $62 \%$ de IU et $66 \%$ de TUC). 
- $\quad$ Bien moins de problèmes communs à IU et TUM $(8$, soit $24 \%$ de UI et $42 \%$ de TUM).

- $\quad$ Encore moins de problèmes communs à TUC et TUM (4, c'est-à-dire $12 \%$ de TUC et $21 \%$ de TUM).

- Très peu de problèmes communs aux 3 méthodes IU, TUC et TUM (4, c'est-à-dire $12 \%$ de IU, $12 \%$ de TUC et $21 \%$ de TUM).

En outre, on peut noter que :

- Les 4 problèmes communs à TUC et TUM ont aussi été diagnostiqués avec IU.

- 17 problèmes (sur 21 problèmes communs) ont été diagnostiqués avec TUC et IU, mais seulement 4 ont également été diagnostiqués avec TUM.

- $\quad 4$ problèmes (sur 8 problèmes communs) ont été diagnostiqués à la fois avec TUM et IU, et 4 avec TUC.

Contrairement aux conclusions de [Bach and Scapin 2011, op. cit.], TUC et TUM ne semblent pas conduire à une plus grande diversité de problèmes d'utilisabilité. Cependant, on observe que TUC et TUM semblent particulièrement efficaces pour le diagnostic des problèmes qui nécessitent un état particulier d'interaction pour être détectables, C'est le cas de la saisie des formulaires, avec TUC et TUM nous avons detectés des problèmes qui bloquent l'utilisateur (par exemple : "Après la saisie des informations de sécurité sociale, les participants cochent la case et clickent sur le bouton «AJOUTER» confondu avec le bouton «ENREGISTRER». Des exemples de recommandations sont également proposées:

R.25: (issue del IU) Placer le bouton «Enregistrer» à proximité de chaque groupe d'items à enregistrer,

R.26: (issue de TUC et TUM) Proposer un bouton doté d'une icône "+ + après les formulaires. Cette symbolique est déjà souvent utilisée dans les systèmes pour l'ajout d'un champ.


médecin » ou « Autre Enfant », etc. »)

D'un autre côté, IU semble aider à l'identification de problèmes basiques, directement observables, souvent liés à la facilité d'apprentissage et d'utilisation.

Une autre analyse a été menée pour tenter de clarifier le rôle respectif des analyses expérimentales (issues des données de comportement observé), des commentaires participants (à leur initiative). On a ainsi relevé, pour chaque problème d'utilisabilité, la source de son diagnostic : analyse (erreurs, temps passé, etc.), commentaire participant, ou les deux. Ceci a concerné uniquement les méthodes TUC et TUM, et non IU qui, étant une inspection (de l'application, indépendamment des utilisateurs), ne se prête pas à une telle analyse.

Globalement (voir Tableau 9), on constate déjà que, d'une part, près de la moitié des diagnostics (36) ont été faits à partir des deux méthodes, puis (29) à partir des commentaires participants, puis (18) à partir des analyses de l'expérimentation. On confirme aussi la part un peu plus élevée de diagnostics pour TUC (48) par rapport à ceux pour TUM (35). 


\begin{tabular}{|l|c|c|c|c|}
\hline Méthode & $\begin{array}{c}\text { Analyse } \\
\text { Expé. }\end{array}$ & $\begin{array}{c}\text { Commentaire } \\
\text { Participant }\end{array}$ & Les deux & Tot. \\
\hline TUC & $\mathbf{1 5}$ & $\mathbf{1 5}$ & $\mathbf{1 8}$ & 48 \\
& $(7+8)$ & $(4+4(2 \times 2)+3+4(2 \times 2))$ & $(4(2 \times 2)+10+4(2 \times 2))$ & \\
\hline TUM & $\mathbf{3}$ & $\mathbf{1 4}$ & $\mathbf{1 8}$ & 35 \\
& $(2+1)$ & $(4+4(2 \times 2)+2+4(2 \times 2)$ & $(5+4(2 \times 2)+5+4(2 \times 4))$ & 83 \\
\hline
\end{tabular}

Tableau 8 : Analyse/Commentaires par Méthode

Examinons plus en détail (voir Tableau 10) les problèmes d'utilisabilité spécifiques et communs aux 3 méthodes (on notera que les totaux ne sont pas équivalents au Tableau 9 précédent car, dans celui-ci, on comptabilise les problèmes, pas leurs instances, contrairement au tableau précédent).

\begin{tabular}{|l|c|c|c|c|}
\hline \multicolumn{1}{|c|}{ Méthode } & $\begin{array}{c}\text { Analyse } \\
\text { Experimentation }\end{array}$ & $\begin{array}{c}\text { Commentaire } \\
\text { Participant }\end{array}$ & Les deux & \multicolumn{1}{c|}{} \\
\cline { 2 - 5 } Spécifiques TUC & $\mathbf{7}$ & $\mathbf{4}$ & $\mathbf{0}$ & 11 \\
\hline Spécifiques TUM & $\mathbf{2}$ & $\mathbf{4}$ & $\mathbf{5}$ & 11 \\
\hline Communs TUC \& TUM & $\mathbf{0}$ & $\mathbf{2}$ & $\mathbf{2}$ & 4 \\
\hline Communs IE \& TUC & $\mathbf{8}$ & $\mathbf{3}$ & $\mathbf{1 0}$ & 21 \\
\hline Communs IE \& TUM & $\mathbf{1}$ & $\mathbf{2}$ & $\mathbf{5}$ & $\mathbf{8}$ \\
\hline Communs TUC/ TUM/ IE & $\mathbf{0}$ & $\mathbf{2}$ & $\mathbf{2}$ & 4 \\
\hline
\end{tabular}

Tableau 9 : Analyse/Commentaires par Méthode et Combinaisons

On constate que les problèmes diagnostiqués à partir des commentaires des participants (ou les deux) prennent une part importante. En effet, comme déjà noté auparavant, dans le scénario avec modification, l'utilisateur assume un rôle plus important, il est plus actif, et parle plus.

On remarque que 5 des problèmes qui ont été diagnostiqués à partir de l'analyse (TUM) sont également commentés par l'utilisateur, différemment de TUC, où aucun des problèmes diagnostiqués par l'analyse n'ont étés commentés. Sur un total de 11 problèmes détectés, 9 ont été commentés par l'utilisateur, tandis que dans TUC seulement 4 sur 11 l'ont été.

Voici un exemple de problème diagnostiqué à partir de l'analyse et du commentaire des participants dans le TUM : La catégorie TRAVAIL a été ciblée de changements de dénomination pour mieux exprimer ce qu'on trouve en son sein et aussi une envie de pouvoir la séparer en deux catégories différentes et indépendantes. Dans ce cas, le commentaire participant permet une meilleure compréhension du problème déjà diagnostiqué par l'analyse. 
Voici un exemple de problème issu exclusivement du commentaire du participant dans le TUM : "On a pu s'apercevoir aussi que le masquage est utilisé aussi comme une façon de "protéger » et "sécuriser » leurs données personnelles. Ils remplissent leurs informations, mais ensuite ils les «cachent» (en les masquant) : «... si j'ai besoin de la visualiser, je sais où je peux la démasquer, mais comme ça elle n'est pas visible ». C'est le cas pour des rubriques comme Dossier Médical, Famille. ». Ce type de commentaire, nous a permis de diagnostiquer le problème de l'utilisateur en rapport à la sécurité de ses données.

Dans cette étude, quelques limites doivent être mentionnées :

- Pour TUC et TUM, la présence de l'expérimentateur a pu entraver l'accès à certaines fonctions du prototype (par exemple, aide en ligne) car il était juste plus simple de demander à l'expérimentateur.

- Sauf dans le scénario de modification, les participants sont restés concentrés sur les tâches, sans explorer davantage. Un suivi des utilisateurs sur des périodes plus longues pourrait être utile. Cela pose des questions techniques (méthodes de recueil, $\operatorname{logs}$, etc.), et méthodologiques ardus, notamment en termes de fouille de données pertinentes sur les intentions des utilisateurs au cours du temps.

- Un ensemble varié de problèmes d'utilisabilité a été identifié. Cependant, il n'y avait aucun contrôle sur les défauts d'utilisabilité résultant (involontairement) de la conception par une entreprise partenaire spécialisée dans les applications egouvernement et les outils de conception. Cela empêche bien sûr de généraliser pleinement à toutes les autres applications de ce type. Dans l'avenir, il serait intéressant d'introduire volontairement un grand nombre de défauts d'utilisabilité sur tous les états de l'application, avec une variation complète des types de problèmes, afin d'avoir un contrôle sur le nombre total de problèmes, leur nature et leur emplacement, ce qui permettrait d'extraire l'ensemble des diagnostics en fonction de chaque technique d'évaluation.

- Seuls la structure de l'information et le contenu du prototype ont été évalués, pas les procédures électroniques associées, qui ne seront mises en œuvre que plus tard dans le projet PIMI.

- En termes d'interaction, les contraintes techniques n'ont pas permis la manipulation directe, notamment pour les changements d'ordre des catégories.

\section{CONCLUSION}

Cet article a présenté un travail de recherche associé à l'évaluation de l'utilisabilité d'un prototype pour la gestion des données personnelles. L'expérience, avec 30 participants (chercheurs, administratifs et étudiants), a suivi 4 scénarios de tâches classiques de tests utilisateurs et 1 scénario permettant l'adaptation de la structure de l'information de prototype et son contenu par les participants.

Les résultats ont permis le diagnostic de 51 problèmes d'utilisabilité et ont abouti à des recommandations pour améliorer la version finale du prototype. Ces recommandations portent sur la lisibilité (couleurs, contrastes, taille, type de police), la navigation, les formulaires (forme et modes d'enregistrement), les modes de modification, la sécurité des informations... 
D'un point de vue méthodologique, nous avons étudié le rôle relatif des deux méthodes d'évaluation utilisées dans l'expérience (TUC et TUM). En outre, les résultats ont également été comparés aux résultats d'une inspection de l'utilisabilité (IU).

Les trois techniques d'évaluation sont tout à fait complémentaires, mais la participation directe des utilisateurs par personnalisation est une méthode additionnelle utile, permettant d'identifier des problèmes d'utilisabilité spécifiques, notamment de nouveaux chemins, diverses utilisations de fonctions, et différentes compréhensions des concepts.

Bien que l'inspection basée sur les critères ergonomiques ait permis de faire un diagnostic général des problèmes d'utilisabilité (plusieurs types et dans de nombreux états de l'interface), le test utilisateur a permis d'identifier des problèmes similaires, mais avec plus de détails, bien que sur un plus petit nombre d'états d'interaction.

Une autre étude récente [Schmettow, Bach, and Scapin, 2012] a aussi montré que l'inspection ergonomique et le test utilisateur permettaient de repérer des problèmes assez disjoints. De plus, il apparaît que la combinaison des deux techniques d'évaluation permet d'identifier $20 \%$ de problèmes de plus.

L'utilisation d'un scénario de personnalisation permet aux utilisateurs de s'exprimer davantage sur le système en indiquant leurs préférences et ainsi de promouvoir une évaluation et une conception plus collaborative.

Dans l'ensemble, la participation active et directe des utilisateurs, en offrant de nouvelles structures, modalités, chemins et fonctions, sont des indices additionnels très riches pour l'évaluation et la conception.

Pour des travaux de recherche futurs, un certain nombre de questions semblent intéressantes à étudier :

- La sécurité et la confiance sont toujours un problème, même si une perception positive semble facilitée par l'implication des utilisateurs dans l'adaptation de la structure et du contenu. Il conviendrait de valider cela pour des e-procédures complètes.

- L'usage réel : il faudra trouver des moyens efficaces de l'évaluer grâce à des outils innovants et intelligents pour la collecte des données utilisateur en temps réel, y compris sur l'évolution des personnalisations au cours du temps.

- La mobilité : le futur système PIMI devrait être également utilisé sur des smartphones et autres tablettes. Une version PIMI de mobilité est en cours d'élaboration, mais sa facilité d'utilisation et la compatibilité avec la version PC, y compris sa plasticité devront être évaluées.

\section{REMERCIEMENTS}

Cette étude a été menée par l'INRIA, contrat ANR-PIMI.

\section{RÉFÉRENCES}

Ardito, C., Costabile, M. F., Desolda, G., Matera, M., Piccinno, A., And Picozzi, M. 2012. Composition of situational interactive spaces by end users: a case for cultural heritage Living history: Proc. 7th Nordic Conference on HCI 2012-10-14 p.79-88. 
BACH, C. AND SCAPIN, D. L. 2010. Comparing Inspections and User Testing for the Evaluation of Virtual Environments, in : International Journal of Human-Computer Interaction, vol. 26, no 8, p. 786-824.

ButtField-Addison, P., Lueg, C., Ellis, C., ANd Manning, J. 2012. Everything goes into or out of the iPad: the iPad, information scraps and personal information management: Proc. of 2012 Australian CHI Conference 2012-11-26 p.61-67.

Cockton, G., Woolrych, A., Hall, L., And Hidemarch, M. 2003, Changing Analysts' Tunes: The Surprising Impact of a New Instrument for Usability Inspection Method Assessment: In Proceedings of People and Computers XVII : Designing for Society, Springer Verlag, pp. 145-162.

CSCW13-1, 2013. Proceedings of the 2013 ACM Conference on Computer Supported Cooperative Work v.1 / Amy Bruckman / Scott Counts / Cliff Lampe / Loren Terveen San Antonio, Texas 2013-02-23 2013-02-27 2013 ACM n.141 p. 1566.

Demunieux, R., Ganneau, V., Calvary, G., And Gegovska, E. 2008. Les interfaces plastiques : premiers retours utilisateurs, évaluations en laboratoire : Ergo-IA 2008. 117-124.

Detraux, C. AND SCAPIN, D. L. 2012. La modification utilisateur comme indice ergonomique évaluatif d'un Espace Personnel d'Information : in Ergo'IHM 2012, Biarritz, France, 17-19 Octobre, 16-23.

DÖRner, C., Yetim, F., PiPEK, V., AND Wulf, V. 2011. Supporting business process experts in tailoring business processes: Interact. Comput. 23, 3, 226-238.

Evequoz, F. AND Lalanne, D. 2009. I thought you would show me how to do it studying and supporting PIM strategy changes : PIM Workshop, ASIS\&T 2009, 3542 .

Gamberini, L., Spagnolli, A., Carradi, N., Jacucci, G., Tusa, G., Mikkola, T., ZAMBONI, L., AND HogGan, E. 2012. Tailoring Feedback to Users' Actions in a Persuasive Game for Household Electricity Conservation : Proceedings of the 2012 International Conference on Persuasive Technology p.100-111.

Gray, W. D. AND SAlzMAN, M. C. 1998, Damaged merchandise? A review of experiments that compare usability evaluation methods Human-Computer Interaction, 13, pp. 203-261.

Hartson, H. R., Andre, T. S. And Williges, R. C., 2001, Criteria for evaluating usability evaluation methods: International Journal of Human-Computer Interaction, 13, pp. 373-410.

HENDERSON, S. 2009. Guidelines for the design of personal document management user interfaces : PIM Workshop, ASIS\&T 2009, pp. 65-72.

HENDERSON, S. AND SRINIVASAN, A. 2009. An empirical analysis of personal digital document structures, Proc. HCI International 2009, Berlin, Heidelberg: SpringerVerlag, pp. 394-403.

Heung-NAm, K., Majdi, R., AND El SADDIK, A. 2013. Tailoring recommendations to groups of users: a graph walk-based approach : Proceedings of the 2013 International Conference on Intelligent User Interfaces 2013-03-19 v.1 p.15-24

HORNBÆK, K. AND FRøKJÆR, E., 2005, Comparing usability problems and redesign proposals as input to practical systems development : In Proceedings of ACM 
Conference on Human Factors in Computing Systems, (New York, NY : ACM Press), pp. 391-400.

ISO/TR 16982. 2002. Ergonomics of human-system interaction - Usability methods supporting human-cenred design. International Standards Organization.

KAY, J. AND, KUMMERFELD, B. 2012. Creating personalized systems that people can scrutinize and control: Drivers, principles and experience. ACM Transactions on Interactive Intelligent Systems 2012-12 v.2 n.4, pp. 24 :1-24 :42.

KolÅs, L. And STAupe, A. 2007. A Personalized E-learning Interface. In : The Proceedings of the international IEEE Conference on Computer as a tool. Eurocon 2007 (pp. 2670-2675). Poland : IEEE Computer Society.

LindROTH, T., AND BERGQUIST, M. 2008. Breadcrumbs of interaction : situating personal information management : Proc. Fifth Nordic Conference on HCI 2008-1010 p.266-273.

Mirri, S., Salomoni, P., AND Prandi, C. 2011. Augment browsing and standard profiling for enhancing web accessibility : Proceedings of the 2011 International Cross-Disciplinary Conference on Web Accessibility (W4A) 2011-03-28 v.2 pp.510

MørCh, A. I., AND MEHANDJIEV, N. D. 2000. Tailoring as collaboration : the mediating tole of multiple representations and application units. Computer Supported Cooperative Work. 9(1) : 75-100.

Nitsche, M., AND NuERnBerger, A. 2012. InFrame-Browsing : Enhancing Standard Web Search: Proc. of Symposium on HCI and Information Retrieval 2012-10-04 (Poster Paper) p.16.

OAKLEY, R. L. AND SAlam, A. F. 2012. Cyber Citizens and Cyber Deviance : Exploring Social and Technical Factors as Antecedents to Cyber Deviance and the Implications for Cyber Citizenship : Proceedings of the 2012 AIS SIGHCI Workshop on HCI Research in MIS 2012-12-16. Paper 7.

Ploderer, B. Smith, W., Howard, S., Pearce, J., And Borland, R. 2012. Things you don't want to know about yourself: ambivalence about tracking and sharing personal information for behaviour change: Proc. of 2012 Australian Computer-Human Interaction Conference 2012-11-26 p.489-492.

RAWASSIZADEH, R. 2012. Towards sharing life-log information with society / Behaviour and Information Technology 2012-11 v.31 n.11 p.1057-1067.

SCAPIN, D. L. LAW, E., BEvan, N. and the WG1 contributors. 2008. Final Report MAUSE COST 294 WG 1: Critical Review and Analysis of Individual UEMs. COST-294 Project MAUSE.SCAPIN,

ScAPin, D. L., Marie-Dessoude, P., Winckler, M., AND Detraux, C. 2011. Personal Information Systems : User Views and Information Categorization : in Proc. Centric2011-4th. Intl. Conf. on Advances in Human-oriented and Personalized Mechanisms, Technologies, and Services, Barcelona, Spain. pp 154-160.

SchmeTtow, M., BACH, C., AND SCAPIN, D. L., 2014. Optimizing Usability Studies by Complementary Evaluation Methods. 2014 Proceedings of the 28th International \{BCS\} Human Computer Interaction Conference, Southport, UK, 9-12 September 2014, pp 110-119. 
Van Kleek, Max G., Styke, W., Schraefel, M. C., Karger, D. 2011. Finders/keepers : a longitudinal study of people managing information scraps in a micro-note tool Reading \& writing : Proc. ACM CHI 2011 2011-05-07 v.1 p.29072916.

VoIT, K., ANDREWS, K. AND SlANY, W. 2009. Why personal information management (PIM) technologies are not widespread : PIM Workshop, ASIS\&T 2009, 60-64.

WINCKLER, M., GAITS, V., Vo, D.-B., FIRMENICH, S., AND Rossi, G. 2011. An approach and tool support for assisting users to fill-in web forms with personal information New frontiers in documentation II : ACM 29th Intl. Conf. on Design of Communication 2011-10-03 p.195-202.

ZHU, L., VAGHI, I., BARRICELI, B. R. 2011. A meta-reflective wiki for collaborative design: Proc. of the 7th International Symposium on Wikis and Open Collaboration (WikiSym'11).ACM, 53-62.

ZimMERMAN, A., AND LORENTZ, A. 2002. LISTEN : a user-adaptive audio-augmented museum guide Special issue on Personalizing Cultural Heritage Exploration. User Modeling and User-Adapted Interaction 2008-11 v.18 n.5 p.389-416.

\begin{tabular}{|l|l|} 
D. L. Scapin est Directeur de Recherche à l'I.N.R.I.A. Il mène \\
depuis 1977 des recherches en ergonomie des logiciels, notamment \\
sur les méthodes d'évaluation et de conception des IHM : par ex. sur \\
les langages de commande; les critères ergonomiques pour les \\
interfaces graphiques, le web, les environnements virtuels; les \\
méthodes et outils de description des tâches, le multimedia et la \\
multimodalité; ainsi que sur le e.gov. et les données personnelles. \\
Auteur de nombreuses publications scientifiques, il est Editeur- \\
Associé pour BIT et relecteur pour de nombreuses revues et \\
conférences. Il est expert auprès de l'ISO et de l'AFNOR, et pour \\
diverses organisations nationales et internationales. Il est ancien \\
président de l'Human Factors Society Europe et de l'AFIHM.
\end{tabular}

Claudia Detraux est Consultante Ergonome / IHM dans les
domaines du Web, applications mobiles, applications métiers à
Bertin Ergonomie. Auparavant elle était Ingénieur Expert à
l'INRIA-Rocquencourt (2011-2013) et Enseignante-Chercheur à
L'Ecole Polytechnique de Marseille (2006-2010). Ses principaux
thèmes de recherche concernent la conception et l'évaluation des
IHM de systèmes d'information et de systèmes critiques,
l'informatique du citoyen l'erreur humaine issue de problèmes
d'interfaces, l'intégration de la connaissance ergonomique. Docteur
en Ingénierie de l'informatique - IHM (2006), de l'UFCG, Brésil ;
Master en Informatique - Ergonomie du logiciel (2002) et Licence
de Designer de Produit (1996), de l'UFPB, Brésil. Elle est membre
de l'AFIHM.

